\title{
19. Chinese livestock farms struggle under COVID-19 restrictions
}

\section{Xiaobo Zhang}

After the COVID-19 outbreak began in December in Hubei Province, many Chinese villages were locked down to control the spread of the disease. As the epidemic has eased, China has only begun to lift some restrictions. The lockdowns have had a significant - and still not well-understood - impact on the agriculture sector. The effective supply of agricultural products forms the foundation for a stable, functioning economy and safeguards people's livelihoods. Thus, keeping agricultural enterprises running is an indispensable economic component in the ongoing battle against the epidemic - yet discussions of the outbreak have thus far devoted very little attention to the challenges they face.

There are two key problems now.

First, livestock farmers face severe pressure from supply and market disruptions, since animals need to eat every day and production cycles are short - daily for dairy, six weeks for chickens, and three months for pigs. In addition, the pork industry is still reeling from the 2019 outbreak of African swine fever that reduced the country's pig herd by more than $40 \%$ and drove up prices.

Second, the arrival of the spring plowing season is putting crop farmers in a bind. They urgently need to return to work. But the outbreak and ongoing control measures present many challenges. While manufacturing and service enterprises can flexibly adjust their production schedules to mitigate losses arising from the epidemic, the agriculture sector waits for no one. The normal phase of spring plowing includes the provision of labor, seed, fertilizer, pesticide, and agricultural machinery, all within a set timeframe. Once smallholder farms miss the necessary services, such as plowing and pollination, during the critical farming season, their income for the entire year will fall.

To understand the operational situation and demands of small, medium, and micro enterprises impacted by the epidemic, the Enterprise Survey for Innovation and Entrepreneurship in China (ESIEC) project team conducted telephone and online follow-up interviews in February with enterprises surveyed over the past three years, which include some in the agriculture sector. The survey probed their work resumption and production situation, the main difficulties they face, their efforts to adapt, demands for appropriate policies, and other issues.

Most Chinese farms are household smallholders and not registered as enterprises. Therefore, our survey does not capture the direct impact on crop farms. But other types of agribusinesses, such as mechanization services, pollination services, fertilizer dealers, and livestock farms, are covered in our sample and provide a broad picture of the impacts. As of February 10, only $24.6 \%$ of agricultural businesses had resumed production. Since these businesses provide key inputs or services, interruptions in their services may negatively impact agricultural production. 
In our analysis, we found that the main issue agricultural enterprises face is disruption of logistics, especially shortages of raw materials and delivery problems. The stress is particularly acute for livestock farmers: $38.5 \%$ of them list "logistics disruption" as the biggest challenge, compared to $35.6 \%$ of all agricultural enterprises, $19.7 \%$ of non-agricultural enterprises, and $18.9 \%$ of the service sector.

Shortages of raw materials - in particular an inadequate supply of feed to livestock farmers - are the main result of these disruptions. While about $60 \%$ of the agricultural enterprises surveyed have encountered such shortages, they are most severe in the livestock farming sector - where feed shortages mean that animals and poultry may starve to death. With the preexisting problems and lingering high prices from the swine fever outbreak, the industry faces a crisis that could lead to more price spikes.

Overall, respondents' two most common complaints were that feed could not be delivered to the farms, and that trucks could not enter villages to collect their products.

The results can be dire. On February 13, for instance, a beekeeper in Sichuan Province committed suicide after his bees starved to death because his truck of beehives was not allowed to travel across regions to provide pollination services as scheduled. The lack of pollination services may lead to lower yields for many crops.

What policies are needed to address these problems? More than other industries, agricultural enterprises and particularly those in the livestock farming sector say they prefer rent reductions, financing support, and especially force majeure certification.

Our survey makes it clear that, apart from the above options, a simpler and more direct demand often expressed by the entrepreneurs is that the lockdown be ended. The Chinese government has already rolled out a series of pertinent measures, including opening a "green channel" for feed, in order to effectively stabilize agricultural production. However, this has not yet been implemented in all areas. While all relief efforts are important, it is even more urgent that these measures be implemented at the grassroots level. While maintaining effective control of the epidemic, it should be of the greatest importance to encourage enterprises in rural areas to return to work.

Given that other countries and regions around the world have also adopted lockdown policies, the survey results suggest that as spring arrives, agricultural enterprises in many places face serious logistics problems, and that livestock farming also faces challenges similar to China's - problems that may require government intervention to avert shortages or price spikes.

Originally published March 26, 2020. 